\title{
Mechanisms Associating Improper Diet and Obesity with Infertility
}

Created by: QKinga Skoracka, Mlwona Krela-kaźmierczak

Version received: 13 May 2020

check for updates

Skoracka, K.; Eder, P.; Łykowska-Szuber, L.; Dobrowolska, A.; Krela-Kaźmierczak, I.

Department of Gastroenterology, Dietetics and Internal Diseases, Poznan University of Medical Sciences, Heliodor Święcicki Hospital, 60-355 Poznań, Poland

A high-fat diet and obesity, the development of which is encouraged by the western lifestyle, can significantly affect male fertility. An inappropriate diet may be directly related to the increase of oxidative stress, but also contribute to the development of obesity, which is associated with the deterioration of fertility, both in terms of generating oxidative stress as well as hormonal or immunological disorders. In obese individuals, disorders on the hypothalamic-pituitary-gonadal axis are observed, as well as elevated oestrogen levels with simultaneous decrease of testosterone, $\mathrm{LH}$ and FSH hormone levels.

Weight loss in obese men seems to be the first, and the most basic step in the treatment of male infertility.

In recent decades there has been a drastic change in society's lifestyle - with a reduction in energy expenditure, particularly in daily physical activity, the consumption of hypercaloric foods with high glycaemic index and high fat content has increased, with a simultaneous low intake of dietary fibre [ㅍ] This, in turn, has resulted in a significant increase in the proportion of obese individuals worldwide -

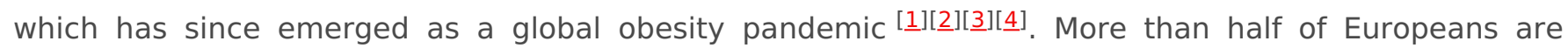
overweight or obese, and men are much more likely to be overweight than women [ $]$.

It is generally accepted that excessive body weight has a negative impact on the body, contributing to the development of diseases, such as diabetes, hypertension, cardiovascular diseases, cancer, sleep apnoea, or osteoarthritis. In fact, the impact of obesity on reproductive functions is also relevant [2][]ㅡ][].

An unhealthy hypercaloric diet, excessive intake of saturated fats and trans-fatty acids, high glycaemic index, and low nutritional density may be directly associated with increased oxidative stress, which constitutes the underlying cause of obesity, intestinal dysbiosis, type 2 diabetes, and insulin resistance []]ㅁ][]ㅛ.

The above-mentioned metabolic disorders are associated with a deterioration of fertility mainly due to the generation of oxidative stress as well as hormonal and immunological disorders [를 $]$. Thus, an increase in white adipose tissue leads to an increase in the production of pro-inflammatory cytokines and reactive oxygen species, as well as the aromatase activity that is responsible for the conversion of testosterone to oestradiol. On the other hand, obese men with type 2 diabetes and insulin resistance are more likely to experience secondary hypogonadism and lower levels of sex hormone-binding protein (SHBG). Moreover, hyperglycaemia has a negative impact on sperm motility and the fertilization process [].

The key mechanism that associates improper diet and obesity with both lower semen quality and an increased risk of infertility is oxidative stress. Moreover, it is currently considered one of the leading causes of male infertility ${ }^{[9]}$, together with the decrease in antioxidant activity and dysfunctional activity 
of mitochondria in spermatozoa. Figure 1 presents the influence of oxidative stress on sperm quality and fertility.

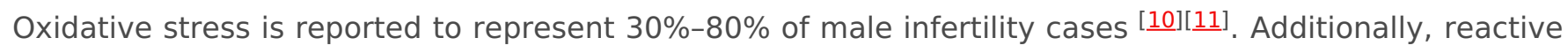
oxygen species (ROS) may impair the motility of spermatozoa and interfere with their ability to connect to the oocyte $[\underline{10]}$.

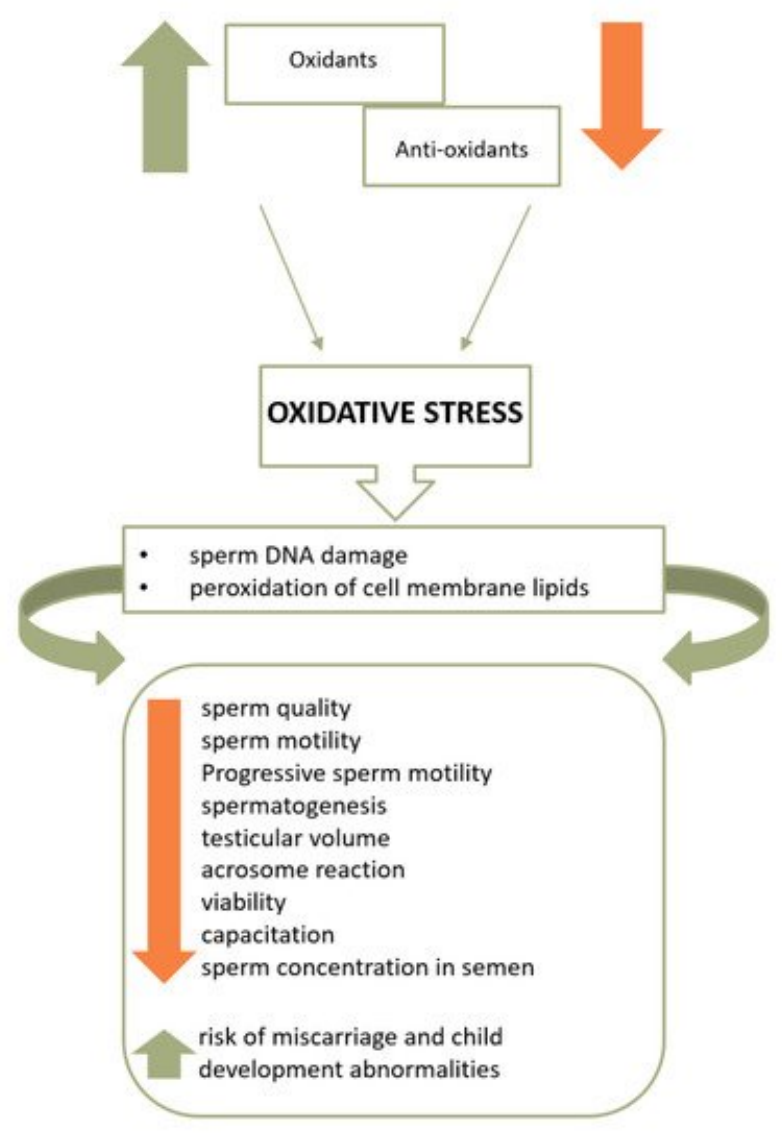

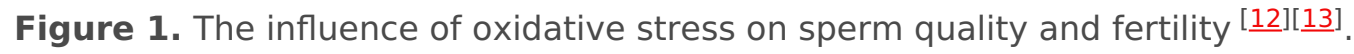

As indicated in Figure 1, cell membrane lipids, proteins, and sperm DNA are damaged ${ }^{[14]}$ once ROS overcomes the sperm antioxidant barrier. As a consequence, the higher the intensity of oxidative stress, the lower the motility, live sperm count, and sperm concentration in the semen, as well as the risk of miscarriage and child development abnormalities [2]][10][15][16]. Moreover, excessive production of ROS is also associated with the deterioration of sperm morphology parameters [1]].

Both the consumption of pro-inflammatory products and a low consumption of high antioxidant potential foods, as well as a high glycaemic index and load in the diet, constitute factors responsible for the increase in oxidative stress. Moreover, glucose metabolism proves to have significant impact on spermatogenesis, whereas hyperglycaemia affects sperm motility and the fertilization process [표] .

In addition, in obese individuals, disorders on the hypothalamic-pituitary-gonads (HPG) axis have been observed. Excess fat tissue results in increased aromatase activity converting testosterone to oestrogen and consequently, to increased oestrogen levels with decreased levels of testosterone and LH hormone, which stimulates steroidogenesis, as well as of $\mathrm{FSH}$, which is responsible for spermatogenesis [미][ㄱ] $[\underline{19}][\underline{20}]$.

Additionally, white adipose tissue (WAT) produces pro-inflammatory cytokines and ROS, the excess of which leads to systemic inflammation and oxidative stress [ $\left.{ }^{[\underline{19}]}\right]$. One of the adipokines produced by WAT is leptin, also referred to as the satiety hormone. Under physiological conditions, leptin inhibits the 
appetite centre and stimulates the secretion of gonadotropic hormones [1]][리]. The plasma concentration of leptin is proportional to the amount of body fat; however, in obese individuals, the phenomenon of leptin-resistance is observed next to hyperleptinemia, in which the hypothalamus does not respond properly to leptin. It is therefore suggested that excessive leptin production may constitute a significant factor in the development of androgen deficiency and deterioration of male reproductive function. Nevertheless, the mechanisms binding leptin to the HPG axis and obesity are still not fully understood and the research results on the role of leptin in infertility development remains unclear [미] $[\underline{22}][\underline{23}]$.

Apart from leptin, the hypothalamic-pituitary-gonads (HPG) axis and spermatogenesis are also affected by other pro-inflammatory cytokines produced in excess by the WAT, such as tumour necrosis factor (TNFa, tumor necrosis factor-a), interleukin-6, chemerin, resistin, or ghrelin [1ㄱ]

Furthermore, excessive body weight is also associated with erectile dysfunction and an increased temperature in the scrotum, which may have an adverse effect on spermatogenesis. Additionally, it also affects the obstructive sleep apnoea syndrome, potentially leading to dysfunction of the HPG axis and disturbed night-time testosterone secretion due to chronic hypoxia and sleep fragmentation [피][16].

In recent decades, the main nutritional model of the developing and developed countries has become the so-called western diet [24][르. Western diet is characterized by a high intake of animal proteins, saturated and trans fatty acids and simple carbohydrates as well as a low supply of dietary fibre and essential unsaturated fatty acids (EFA). Additionally, it is a hypercaloric diet with low nutritional density and pro-inflammatory character ${ }^{[25]}$. It is clear that with the spread of the Western diet model, the parameters evaluating semen quality have deteriorated [리를].

A diet rich in processed and, according to some sources, red meat, fatty dairy, coffee, alcohol, sweet drinks and sweets, potatoes, and simultaneously deficient in whole-grain products, vegetables and fruits, poultry, fish and seafood, nuts, and lean dairy is associated with poorer semen parameters and reduced fertility [18][27][14][ㄹ] . Characteristics of a diet negatively affecting fertility and its proposed modifications is presented in Figure 2.

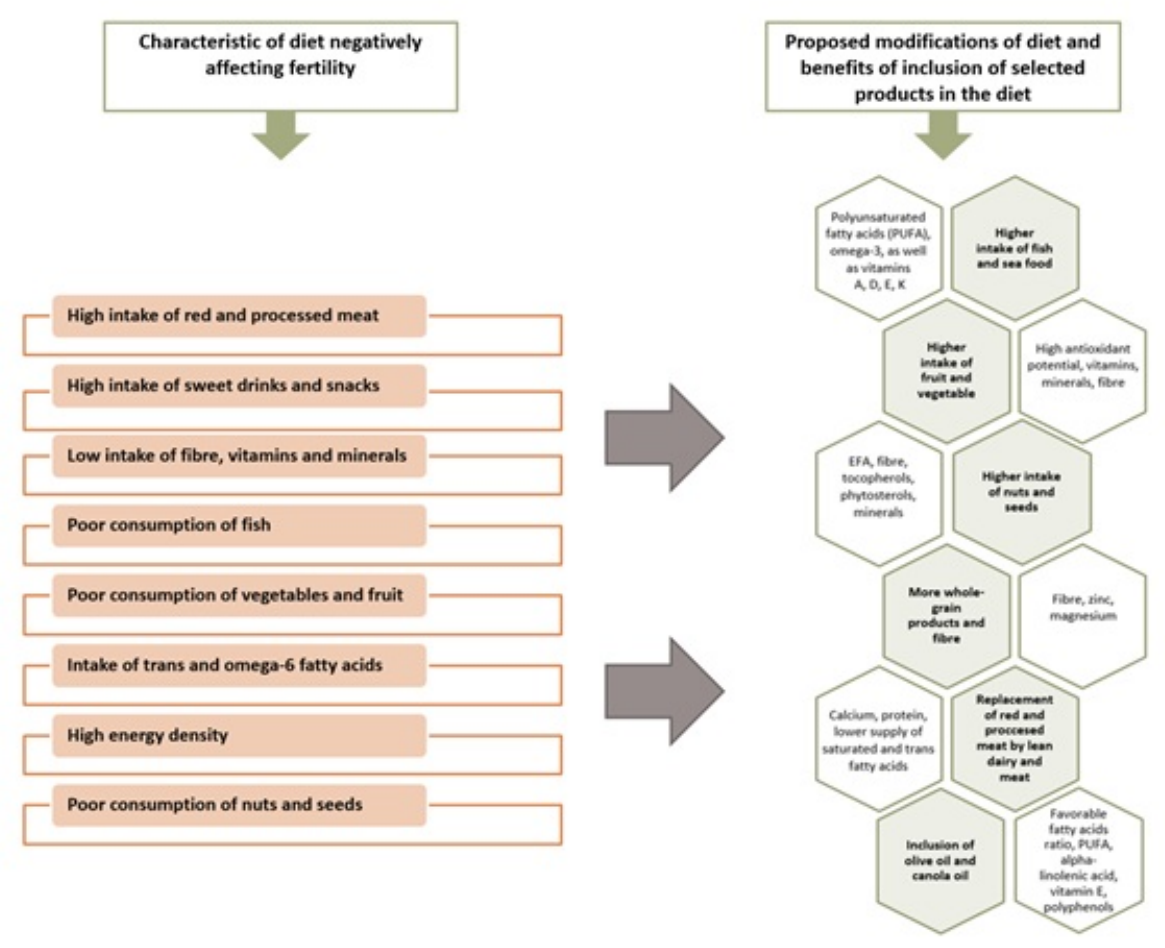

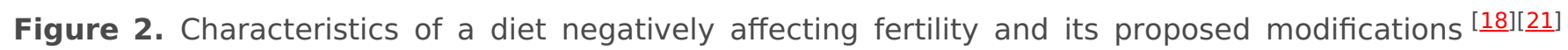
[27]. 
Research studies indicate that healthy dietary models clearly correlate with better sperm quality and a lower possibility of abnormalities in such parameters as sperm quantity, concentration, and motility, as

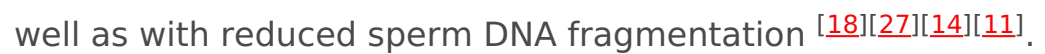

The recommended dietary standard is a diet rich in raw vegetables and fruit, whole-grain, and fibre-rich products, instead of products based on purified flour. Olive oil, oily sea fish from a reliable source, nuts, seeds and stones, and avocados are good sources of unsaturated fats, which can make up to $35 \%$ of the calorific value in the diet. Therefore, a good source of protein is lean poultry and low-fat dairy products, legumes, fish, and seafood [1]ㅛㅇㅛ.

Modification of lifestyle, particularly with regard to the diet, seems to be indispensable with regard to male infertility associated with semen quality.

\section{References}

1. Jill A. Attaman; Thomas L. Toth; Jeremy Furtado; Hannia Campos; Russ Hauser; Jorge E. Chavarro; Dietary fat and semen quality among men attending a fertility clinic. Human Reproduction 2012, 27, 1466-1474, 10.1093/humrep/des065.

2. Renata Walczak-Jedrzejowska; Jan Karol Wolski; Jolanta Slowikowska-Hilczer; The role of oxidative stress and antioxidants in male fertility. Central European Journal of Urology 2013, 66, 60-67, 10.5173/ceju.2013.01.art19.

3. Emily J. Willingham; Environmental Review: Trenbolone and Other Cattle Growth Promoters: Need for a New RiskAssessment Framework. Environmental Practice 2006, 8, 58-65, 10.1017/S1466046606060042.

4. D. M. Henricks; S. L. Gray; J. J. Owenby; B.R. Lackey; Residues from anabolic preparations after good veterinary practice.. APMIS 2001, 109, S345-S356, 10.1111/j.1600-0463.2001.tb05786.x.

5. Myriam C. Afeiche; Audrey J. Gaskins; Paige L. Williams; Thomas L. Toth; Diane L. Wright; Cigdem Tanrikut; Russ Hauser; Jorge E. Chavarro; Processed meat intake is unfavorably and fish intake favorably associated with semen quality indicators among men attending a fertility clinic.. The Journal of Nutrition 2014, 144, 1091-8, 10.3945/jn.113.190173.

6. James Varani; Healthful Eating, the Western Style Diet and Chronic Disease. Approaches in Poultry, Dairy \& Veterinary Sciences 2017, 1, 3, 10.31031/apdv.2017.01.000511.

7. Thomas Chambers; Richard A. Anderson; The impact of obesity on male fertility. Hormones 2015, 14, 563-8, 10.14310/horm.2002.1621.

8. Albert Salas-Huetos; Nancy Babio; Douglas T. Carrell; Mònica Bulló; Jordi Salas-Salvadó; Adherence to the Mediterranean diet is positively associated with sperm motility: A cross-sectional analysis. Scientific Reports 2019, 9 , 3389, 10.1038/s41598-019-39826-7.

9. Sedigheh Ahmadi; Reihane Bashiri; Akram Ghadiri-Anari; Azadeh Nadjarzadeh; Nutrition and Food Security Research Center, Shahid Sadoughi University of Medical Sciences, Yazd, Iran; Diabetes Research Center, Shahid Sadoughi University of Medical Sciences, Yazd, Iran; Antioxidant supplements and semen parameters: An evidence based review. International Journal of Reproductive BioMedicine (IJRM) 2016, 14, 729-736, 10.29252/ijrm.14.12.729.

10. Marian G Showell; Julie Brown; Anusch Yazdani; Marcin T Stankiewicz; Roger J Hart; Rebecca Mackenzie-Proctor; Antioxidants for male subfertility. Cochrane Database of Systematic Reviews 2014, 12, CD007411, 10.1002/14651858.cd007411.pub3.

11. Elena Ricci; S. Al-Beitawi; S. Cipriani; Alessandra Alteri; Francesca Chiaffarino; M. Candiani; S. Gerli; P. Viganó; F. Parazzini; Dietary habits and semen parameters: a systematic narrative review. Andrology 2017, 6, 104-116, 10.1111/andr.12452.

12. Walczak-J ,edrzejowska, R.; Oxidative stress and male infertility. Part I: Factors causing oxidative stress in semen. Adv. Androl. Online 2015, 2, 5-15.

13. Shilpa Bisht; Muneeb Faiq; Madhuri Tolahunase; Rima Dada; Oxidative stress and male infertility. Nature Reviews Urology 2017, 14, 470-485, 10.1038/nrurol.2017.69.

14. Albert Salas-Huetos; Emma R. James; Kenneth I. Aston; Timothy G. Jenkins; Douglas T. Carrell; Diet and sperm quality: Nutrients, foods and dietary patterns.. Reproductive Biology 2019, 19, 219-224, 10.1016/j.repbio.2019.07.005.

15. Leszek Bablok; Wojciech Dziadecki; Iwona Szymusik; Slawomir Wolczynski; Rafal Kurzawa; Leszek Pawelczyk; Piotr Jedrzejczak; Wojciech Hanke; Pawel Kaminski; Miroslaw Wielgos; et al. Patterns of infertility in Poland - multicenter study.. Neuro endocrinology letters 2011, 32, 799-804.

16. Yue Liu; Zhide Ding; Obesity, a serious etiologic factor for male subfertility in modern society. Reproduction 2017, 154, R123-R131, 10.1530/rep-17-0161.

17. Piotr Jedrzejczak; Monika Fraczek; Anna Szumala-Kakol; Grazyna Taszarek-Hauke; Leszek Pawelczyk; Maciej Kurpisz; 
Consequences of semen inflammation and lipid peroxidation on fertilization capacity of spermatozoa in in vitro conditions. International Journal of Andrology 2005, 28, 275-283, 10.1111/j.1365-2605.2005.00547.x.

18. Albert Salas-Huetos; Monica Bulló; Jordi Salas-Salvadó; Dietary patterns, foods and nutrients in male fertility parameters and fecundability: a systematic review of observational studies. Human Reproduction Update 2017, 23, 371-389, 10.1093/humupd/dmx006.

19. Mohamed Ahmed Abd El Salam; Obesity, An Enemy of Male Fertility: A Mini Review. Oman Medical Journal 2018, 33, 3-6, 10.5001/omj.2018.02.

20. James R. Craig; Timothy G. Jenkins; Douglas T. Carrell; James M. Hotaling; M.S.; Obesity, male infertility, and the sperm epigenome. Fertility and Sterility 2017, 107, 848-859, 10.1016/j.fertnstert.2017.02.115.

21. Barbara E. Kahn; Robert E. Brannigan; Obesity and male infertility. Current Opinion in Urology 2017, 27, 441-445, 10.1097/mou.0000000000000417.

22. Khodamoradi, K.; Parmar, M.; Khosravizadeh, Z.; Kuchakulla, M.; Manoharan, M.; Arora, H; The role of leptin and obesity on male infertility.. y. Curr. Opin. Urol. 2020, 30, 334-339, 10.1097/MOU.0000000000000762..

23. Zhang, J.; Gong, M.; Review of the role of leptin in the regulation of male reproductive function. Andrologia 2018, 50, e12965, 10.1111/and.12965.

24. A. Sansone; Carla Di Dato; Cristina De Angelis; Davide Menafra; C. Pozza; Rosario Pivonello; A. M. Isidori; Daniele Gianfrilli; Smoke, alcohol and drug addiction and male fertility.. Reproductive Biology and Endocrinology 2018, 16, 3, 10.1186/s12958-018-0320-7.

25. Kyle Hart; Nick Tadros; The role of environmental factors and lifestyle on male reproductive health, the epigenome, and resulting offspring. Panminerva Medica 2019, 61, 187-195, 10.23736/s0031-0808.18.03531-0.

26. Angela Alamo; Rosita A. Condorelli; Laura M. Mongioì; Rossella Cannarella; Filippo Giacone; Vittorio Calabrese; Sandro La Vignera; Aldo E. Calogero; Environment and Male Fertility: Effects of Benzo- $\alpha$-Pyrene and Resveratrol on Human Sperm Function In Vitro.. Journal of Clinical Medicine 2019, 8, 561, 10.3390/jcm8040561.

27. Ladan Giahi; Shayan Mohammadmoradi; Aida Javidan; Mohamad Reza Sadeghi; Nutritional modifications in male infertility: a systematic review covering 2 decades. Nutrition Reviews 2015, 74, 118-130, 10.1093/nutrit/nuv059.

\section{Keywords}

anti-oxidants; diet; male infertility; nutritional model; semen quality; obesity; hypercaloric diet; improper diet (c) 2020 by the author(s). Distribute under a Creative Commans CC BY license 